\title{
Epigenetic modifications of prostate-derived Ets transcription factor in breast cancer cells
}

\author{
YAMINI SABHERWAL ${ }^{1,2}$, NITIN MAHAJAN ${ }^{1,2}$ and MING ZHANG ${ }^{1,2}$ \\ ${ }^{1}$ Department of Molecular Pharmacology and Biological Chemistry, Northwestern University; \\ ${ }^{2}$ The Robert H. Lurie Comprehensive Cancer Center of Northwestern University, Chicago, IL 60611, USA
}

Received May 30, 2013; Accepted July 5, 2013

DOI: 10.3892/or.2013.2661

\begin{abstract}
The importance of epigenetic alterations such as DNA methylation, histone modification and nucleosome remodeling in breast cancer is well established. Epigenetic alterations are reversible, and much research has been focused on understanding these alterations with the aim of developing effective therapies. Prostate-derived Ets factor (PDEF) is a member of the Ets family of transcription factors and has long been under investigation for its key role in tumor development and progression. To date, no studies have been conducted to elucidate the epigenetic modifications of PDEF in cancer progression. Using breast and prostate cancer cells, we investigated the effect of the methylation inhibitor 5' azacytidine (AZA) on the expression of PDEF in these cells. The inhibition of methylation observed was specific to breast cancer cells as experiments with prostate cells did not exhibit any significant change. Notably, the expression of p21, a cyclin-dependent kinase (CDK) inhibitor 1 and also a target gene of PDEF, was found to be positively correlated with PDEF expression following 5'AZA treatment. Inhibition of methylation led to a decrease in the proliferation rate of MDA-MB-468 cells as revealed by MTT proliferation assay. Other epigenetic alterations such as histone modifications were not observed in these breast cancer cells following treatment with specific HDAC inhibitors. Our data suggest the possibility of epigenetic modification of PDEF due to DNA methylation and involvement of the cell cycle inhibitor p21. Future studies on the epigenetic alterations of PDEF in correlation with p21 or other targets may facilitate the development of effective therapies for the treatment of breast cancer.
\end{abstract}

\section{Introduction}

Epigenetic alterations have long been implicated in both the development and progression of human cancer (1). Epigenetic

Correspondence to: Professor Ming Zhang, Department of Molecular Pharmacology and Biological Chemistry, Northwestern University, 303 E. Superior Street, Chicago, IL 60611, USA

E-mail:m-zhang@northwestern.edu

Key words: epigenetics, prostate-derived Ets factor, p21, DNA methylation, breast cancer changes such as DNA methylation, histone modification and nucleosome remodeling refer to stable alterations in gene expression with no underlying modifications in the genetic sequence itself (2). In contrast to genetic mutations, epigenetic alterations are intrinsically reversible (3) and hence, much research has been focused on understanding the alterations with the aim to develop effective therapies. DNA methylation is the foremost type of epigenetic modification and is well characterized in human breast cancer (4). Breast cancer is one of the leading causes of death in the US and an increasing number of cases per year are reported. Thus, there is an urgent need to design effective therapies to overcome this disease (5).

Ets family members are characterized by an evolutionarily conserved DNA binding domain called the Ets domain and control key cellular processes, including proliferation, differentiation and apoptosis (6-10). hPDEF (prostate-derived Ets factor/prostate-specific Ets or PSE), is one Ets family member in particular that has been intensely investigated for its role in cancer development and progression. Initial reports indicate that PDEF acts as an oncogene (11-13). However, more recent studies suggest that PDEF possesses a tumor-suppressing function $(12,14,15)$. Despite these findings, it is still not known how PDEF suppresses tumor progression. Most of the research conducted to date has used in vitro studies or correlative immunohistochemical analytic methods to evaluate the role of PDEF in cancer progression $(11,12)$.

We undertook the present study, considering the importance of epigenetic alterations in understanding breast cancer and the lack of studies involving epigenetic modifications of PDEF in breast cancer. Using the breast cancer cell line MDA-MB-468, we provide evidence that PDEF undergoes epigenetic modifications such as DNA methylation and is positively correlated with the expression of its target gene p21. These findings provide further understanding of the mechanisms of breast cancer progression and facilitate the evaluation of options for designing effective therapies to treat breast cancer.

\section{Materials and methods}

Chemicals. Methylation inhibitor 5-AZA-2'-deoxycytidine (A3656; Sigma) and various HDAC inhibitors trichostatin A (T-1952; Sigma), nicotinamide (N0636; Sigma), valproic acid (P4543; Sigma), sodium butyrate (B5887; Sigma) and SAHA- 
(auberoyl bis-hydroxamic acid) (GR323-0100; Biomol) were used.

Cell culture. The human MCF-7, MDA-MB-468 and MDA-MB-231 breast cancer cell lines were maintained in Dulbecco's modified Eagle's medium (DMEM) (Invitrogen) and the CWR22rv1 and PC3 prostate cancer cell lines were maintained in RPMI-1640 medium (Invitrogen) supplemented with 5\% fetal bovine serum (HyClone Laboratories) and $1 \%$ penicillin/streptomycin at $37^{\circ} \mathrm{C}$ with $5 \% \mathrm{CO}_{2}$.

Isolation of protein following treatment with the methylation and HDAC inhibitors. Following treatment with either the methylation inhibitor (5'AZA) or the HDAC inhibitors (TSA, SAHA, $\mathrm{NAD}^{+}$, VPA and $\mathrm{NaB}$ ) monolayers of cell were washed twice with ice cold PBS, and then cell lysates were prepared using RIPA buffer with protease inhibitor mixture (Thermo Fisher Scientific). Cellular debris was cleared by centrifugation, and the protein concentration was determined using the BCA protein assay kit (Pierce).

Western blot analysis. Equal amounts of protein lysates were separated by $10 \%$ SDS-PAGE, transferred to a PVDF membrane (GE Healthcare) and subsequently blotted with the anti-PDEF antibody (15), actin (A2066; Sigma) and acetyl H4 (H9164; Sigma). HRP-labeled goat anti-rabbit polyclonal antibody was used as a secondary antibody, and proteins were visualized with an enhanced chemiluminescence substrate kit (Pierce). Actin and $\mathrm{H} 4$ were used as the loading control as required.

Isolation of RNA and RT-PCR. Total RNA was isolated from the cell lines after treatment with 5'AZA $(1 \mu \mathrm{M})$ or TSA $(0.5 \mu \mathrm{M})$ for $24 \mathrm{~h}$ using TRIzol reagent (Invitrogen) according to the manufacturer's instructions. A two-step RT-PCR was used to analyze the mRNA expression of PDEF (15). cDNA was created using oligo(dT) primer and the Moloney murine leukemia virus reverse transcriptase (MMLV-RT) enzyme according to the manufacturer's instructions (Invitrogen). Standard PCR techniques were then conducted with genespecific primers.

3-(4,5-Dimethylthiazolyl-2)-2,5-diphenyltetrazolium bromide (MTT) in vitro proliferation assay. As previously described (16), MDA-MB-468 cells were seeded at 1,000 cells/well in a 96-well dish and allowed to grow at $37^{\circ} \mathrm{C}$ with $5 \% \mathrm{CO}_{2}$. The following day cells were treated with the methylation inhibitor 5-AZA-2'-deoxycytidine at one single dose of $1 \mu \mathrm{M}$. Growth was assayed for 5 days; every day $10 \mu \mathrm{l} /$ well of MTT reagent $(5 \mathrm{mg} / \mathrm{ml})$ was added and incubation was carried out at $37^{\circ} \mathrm{C}$ with $5 \% \mathrm{CO}_{2}$ for $3 \mathrm{~h}$. The medium was aspirated, and $100 \mu \mathrm{l}$ of DMSO was added and mixed until a purple color formed. The cell samples were measured using a plate reader at $570 \mathrm{~nm}$. All experiments were conducted in triplicates.

Statistical analysis. All the experiments were carried out for minimum of three times or as mentioned. Statistical analysis was based on a minimum of three replicates using Microsoft Office Excel. The results were considered significant when the P-value was $<0.05$.

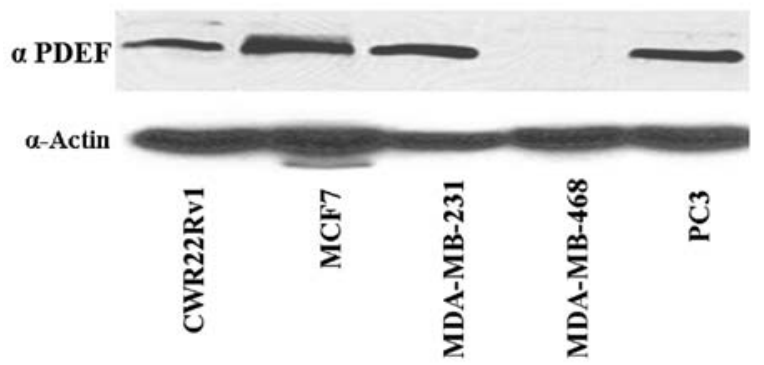

Figure 1. PDEF protein expression analysis of prostate and breast cancer cell lines. Protein expression analysis of CWR22Rv1, MCF7, MDA-MB-231, MDA-MB-468 and PC3 cells with the anti-PDEF antibody.

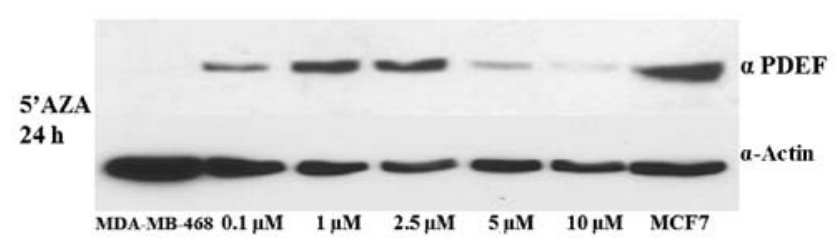

Figure 2. PDEF protein expression analysis of MDA-MB-468 cells following treatment with different concentrations of 5'AZA using an anti-PDEF antibody. Actin was used as the loading control.

\section{Results}

Expression levels of PDEF in the different breast and prostate cancer cell lines. In order to investigate the epigenetic alterations of PDEF, we analyzed the expression of PDEF protein in various breast (MCF7, MDA-MB-231 and MDA-MB-468) and prostate (CWR22Rv1 and PC3) cancer cell lines. All of the cell lines tested expressed PDEF protein except for the breast cancer cell line MDA-MB-468 (Fig. 1). Observation of undetectable PDEF protein expression in MDA-MB-468 human breast cancer cells encouraged us to use these cells as the cellular model for further epigenetic studies.

Inhibition of DNA methylation enhances PDEF expression in MDA-MB-468 cells. To further investigate the epigenetic modifications of PDEF, we treated the MDA-MB-468 cells with a DNA methylation inhibitor 5'AZA. 5'AZA has been widely used in epigenetic research as an inhibitor that blocks DNA methylation to occur (17). Dose-dependent studies for $24 \mathrm{~h}$ revealed that expression of PDEF was enhanced at a dose of $0.1 \mu \mathrm{M}$ of 5'AZA up to a dose of $2.5 \mu \mathrm{M}$ (Fig. 2). We also studied the effect of 5'AZA treatment at the same doses in CWR22Rv1, a prostate cancer cell line, which exhibits weak PDEF protein expression (Fig. 1, lane 1) in comparison to the other cell lines used in the study. CWR22Rv1 cells did not show any significant change following treatment with the methylation inhibitor 5'AZA at the same 24-h time-point (Fig. 3). HDAC inhibitors such as TSA, SAHA, sodium butyrate, valproic acid and nicotinamide are able to cause epigenetic changes and regulate gene expression (18-20). However, we did not observe any significant effect of these HDAC inhibitors on PDEF gene expression in the MDA-MB-468 and CWR22Rv1 cells at different times and dosages (data not shown). These observations clearly indicate that PDEF undergoes DNA methylation as an epigenetic alteration in breast cancer cells. 


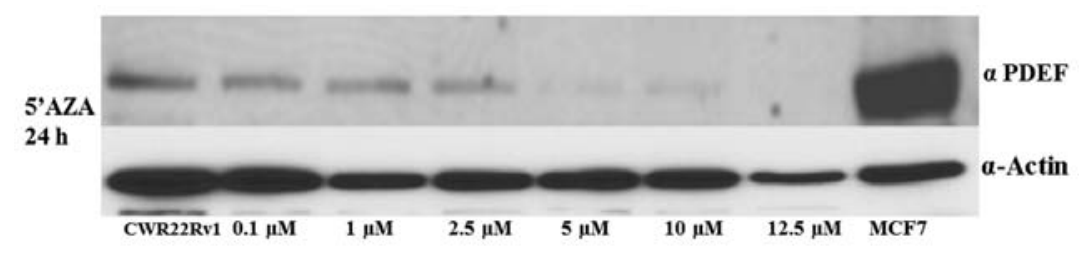

Figure 3. PDEF protein expression analysis in CWR22Rv1 prostate cells following treatment with different concentrations of 5'AZA at $24 \mathrm{~h}$ using the antiPDEF antibody. Actin was used as a loading control. MCF7 serves as a control for PDEF expression.

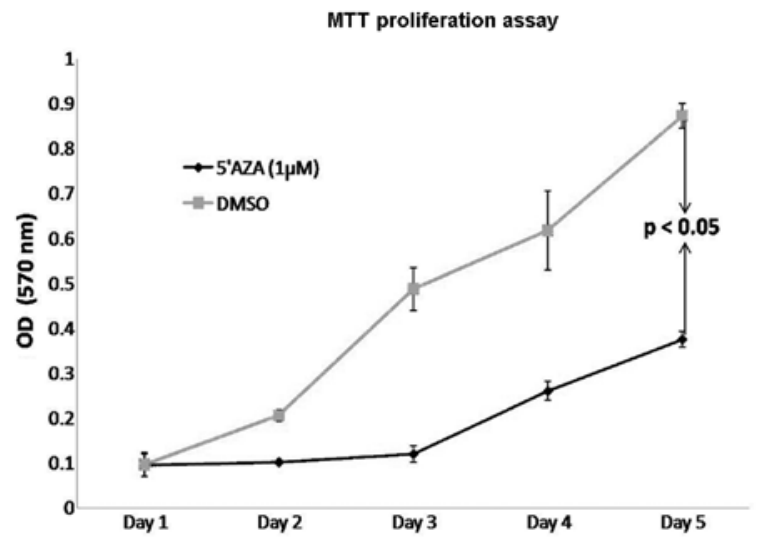

Figure 4. MTT proliferation assay of MDA-MB-468 cells with the MTT reagent following treatment with a single dose of $1 \mu \mathrm{M}$ 5'AZA or DMSO on day 1 . The proliferation rate was calculated for 5 days.

5'AZA treatment significantly inhibits the proliferation rate of $M D A-M B-468$ cells in vitro. To further investigate the functional effects of 5'AZA treatment on cell proliferation in vitro, MDA-MB-468 breast cancer cells were treated with 5'AZA. The cells were treated with 5'AZA at a concentration of $1 \mu \mathrm{M}$, and the proliferation rate was analyzed by MTT assay for a 5-day time period. As shown in Fig. 4, 5'AZA treatment led to a significant decrease in the proliferation rate of MDA-MB468 cells when compared to the controls (DMSO-treated cells). These data indicate that PDEF expression may interfere with the epigenetic modifications such as DNA methylation of mammary tumor cells to decrease or slow the growth or proliferation rate of these cells.

Analysis of the protein level of the cell cycle inhibitor $p 21$ and its correlation with PDEF expression. PDEF controls cell proliferation and mammary tumor progression by transcriptional regulation of the cell cycle inhibitor p21/CIP1 (15). To determine the correlation of PDEF and its target gene p21, expression of p21 was determined in MDA-MB-468 cells following 5'AZA treatment. As shown in Fig. 5A, an increased expression of $\mathrm{p} 21$ was observed following the same expression pattern as that of PDEF. The p21 protein level was increased following 5'AZA treatment at a range of doses from 0.1 to $2.5 \mathrm{mM}$ (Fig. 5B).

\section{Discussion}

PDEF, a member of the Ets family, has been studied intensively since its initial discovery in 2000 (13). The role of PDEF in normal growth and development as well as in disease states
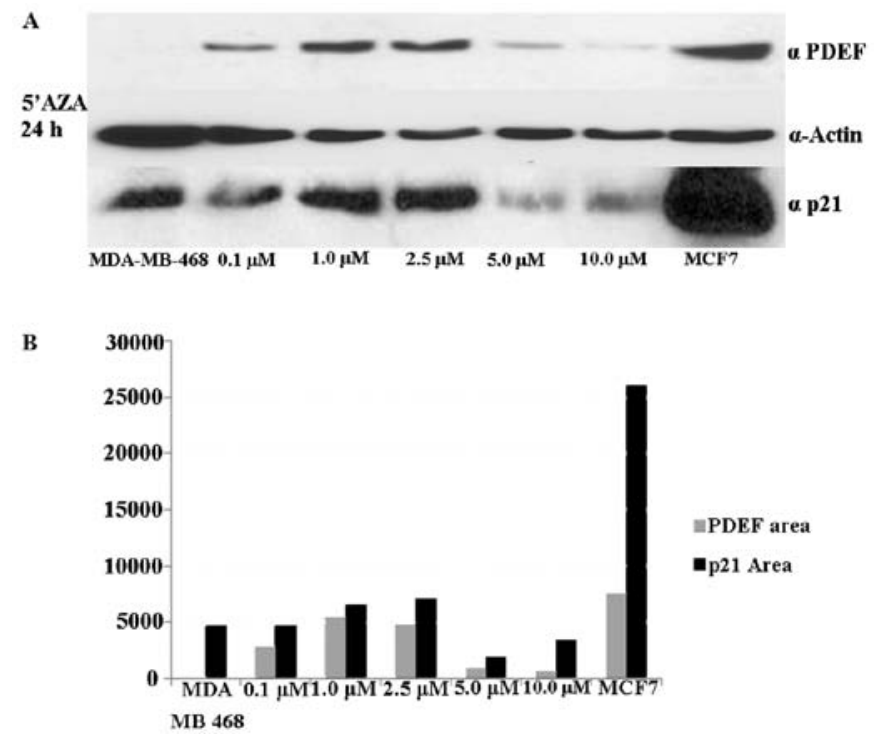

Figure 5. Analysis of PDEF and target gene p21 protein expression in MDA-MB-468 cells following treatment with different concentrations of 5'AZA. (A) PDEF protein expression analysis of MDA-MB-468 cells treated with different concentrations of 5'AZA at a 24-h time-point. (Same as panels in Fig. 2 with the addition of anti-p21). The same blot was probed with the antip21 antibody to assess the levels of cell cycle inhibitor p21 (panel 3). MCF7 serves as a control for PDEF and p21 expression. (B) Bar graph representation of the levels of PDEF and p21 protein expression.

such as cancer is well established $(6-8,10)$. We previously demonstrated a tumor-suppressive role of PDEF in mammary tumor progression and demonstrated the transcriptional regulation of cell cycle inhibitor p21/CIP1 by PDEF (15). In another study, using iTRAQ labeling and proteomic analysis methods the importance of PDEF was established in prostate cancer cells. We demonstrated an association between stathmin, a microtubule-associated protein and PDEF in prostate cancer progression (16).

Epigenetic silencing of a gene can be reversible and therefore, can reactivate gene expression. In the present study, we provide evidence for the epigenetic modifications of PDEF in an in vitro experimental system. After examining various breast and prostate cancer cell lines, we found that only MDA-MB-468 cancer cell line showed no detectable PDEF protein expression (Fig. 1). Thus, we considered the MDA-MB-468 cancer cell line suitable for studying the epigenetic modifications of PDEF gene expression. The MDA-MB-468 cell line was treated with the well-known DNA methylation inhibitor 5'AZA. 5'AZA has been used in many studies and has also been used in cancer therapy $(21,22)$. We observed a dose-dependent enhancement in the PDEF protein 
expression in MDA-MB-468 cells following 5'AZA treatment (Fig. 2). However, with higher concentrations of 5'AZA treatment the PDEF protein expression was reduced. One of the reasons for this reduction could be ubiquitin degradation or 5'AZA toxicity to the cells at higher doses. Notably, DNA demethylation of PDEF was found to be more specific to the MDA-MB-468 breast cancer cell line, as DNA demethylation in the prostate cancer cell line CWR22Rv1 did not show any significant effects (Fig. 3). We further analyzed other epigenetic modifications such as histone acetylation or deacetylation in both the MDA-MB-468 human breast cancer cell line and CWR22Rv1 human prostate cancer cells and did not see any significant changes (data not shown). PDEF in the present study only displayed DNA methylation alteration specific to MDA-MB-468 breast cancer cells. This clearly suggests that in a mammary tumor in vitro experimental design, PDEF exhibited DNA methylation modification.

PDEF has been shown to be associated with many different target genes, such as p21/CIP1, maspin, survivin, p62 and PSA (23-28), to exert its effects based on the co-receptors present and the internal cell environment. In the present study, with human breast cancer cells, PDEF acted as an inhibitor of cell proliferation (Fig. 4). DNA methylation inhibitors have already been used in clinical trials as single-molecule agents and in combinatorial therapies (29-31). The fact that PDEF undergoes DNA methylation reveals the importance to study the underlying mechanism of this epigenetic alteration and the role of PDEF and its correlation with p21 as a target gene.

In conclusion, this is the first report to demonstrate that PDEF undergoes DNA methylation in breast cancer cells. The present study provides insight into the mechanisms involved in the DNA methylation of PDEF. Our report unravels the possibility of further research into the epigenetic DNA methylation of PDEF and the target genes involved in this process in the aim of designing effective and specific PDEF cancer therapeutics.

\section{Acknowledgements}

The authors would like to thank Dr Asish K. Ghosh and Dr George P. Tuszynski for their helpful discussions and comments. The present study was supported by a Northwestern Prostate Cancer SPORE pilot project fund to M.Z.

\section{References}

1. Lustberg MB and Ramaswamy B: Epigenetic therapy in breast cancer. Curr Breast Cancer Rep 3: 34-43, 2011.

2. Yang X, Yan L and Davidson NE: DNA methylation in breast cancer. Endocr Relat Cancer 8: 115-127, 2001.

3. Connolly R and Stearns V: Epigenetics as a therapeutic target in breast cancer. J Mammary Gland Biol Neoplasia 17: 191-204, 2012.

4. Locke WJ and Clark SJ: Epigenome remodelling in breast cancer: insights from an early in vitro model of carcinogenesis. Breast Cancer Res 14: 215, 2012.

5. Siegel R, DeSantis C, Virgo K, et al: Cancer treatment and survivorship statistics, 2012. CA Cancer J Clin 62: 220-241, 2012.

6. Oikawa T: ETS transcription factors: possible targets for cancer therapy. Cancer Sci 95: 626-633, 2004.

7. Galang CK, Muller WJ, Foos G, Oshima RG and Hauser CA: Changes in the expression of many Ets family transcription factors and of potential target genes in normal mammary tissue and tumors. J Biol Chem 279: 11281-11292, 2004.
8. Hsu T, Trojanowska M and Watson DK: Ets proteins in biological control and cancer. J Cell Biochem 91: 896-903, 2004.

9. Li R, Pei H and Watson DK: Regulation of Ets function by protein - protein interactions. Oncogene 19: 6514-6523, 2000.

10. Seth A and Watson DK: ETS transcription factors and their emerging roles in human cancer. Eur J Cancer 41: 2462-2478, 2005.

11. Ghadersohi A and Sood AK: Prostate epithelium-derived Ets transcription factor mRNA is overexpressed in human breast tumors and is a candidate breast tumor marker and a breast tumor antigen. Clin Cancer Res 7: 2731-2738, 2001.

12. Sood AK, Saxena R, Groth J, et al: Expression characteristics of prostate-derived Ets factor support a role in breast and prostate cancer progression. Hum Pathol 38: 1628-1638, 2007.

13. Yamada N, Tamai Y, Miyamoto H and Nozaki M: Cloning and expression of the mouse Pse gene encoding a novel Ets family member. Gene 241: 267-274, 2000.

14. Ghadersohi A, Odunsi K, Lele S, et al: Prostate derived Ets transcription factor shows better tumor-association than other cancer-associated molecules. Oncol Rep 11: 453-458, 2004.

15. Schaefer JS, Sabherwal Y, Shi HY, et al: Transcriptional regulation of p21/CIP1 cell cycle inhibitor by PDEF controls cell proliferation and mammary tumor progression. J Biol Chem 285: 11258-11269, 2010.

16. Sabherwal Y, Mahajan N, Helseth DL, Gassmann M, Shi H and Zhang M: PDEF downregulates stathmin expression in prostate cancer. Int J Oncol 40: 1889-1899, 2012.

17. Ghoshal K and Bai S: DNA methyltransferases as targets for cancer therapy. Drugs Today 43: 395-422, 2007.

18. Acharya MR, Sparreboom A, Venitz J and Figg WD: Rational development of histone deacetylase inhibitors as anticancer agents: a review. Mol Pharmacol 68: 917-932, 2005.

19. Matsuzaki Y, Sowa Y, Hirose T, Yokota T and Sakai T: Histone deacetylase inhibitors: promising agents for 'gene-regulating chemoprevention' and 'molecular-targeting prevention' of cancer. Environ Health Prev Med 8: 157-160, 2003.

20. Ropero S and Esteller M: The role of histone deacetylases (HDACs) in human cancer. Mol Oncol 1: 19-25, 2007.

21. Braiteh F, Soriano AO, Garcia-Manero G, et al: Phase I study of epigenetic modulation with 5-azacytidine and valproic acid in patients with advanced cancers. Clin Cancer Res 14: 6296-6301, 2008.

22. Karahoca M and Momparler RL: Pharmacokinetic and pharmacodynamic analysis of 5-aza-2'-deoxycytidine (decitabine) in the design of its dose-schedule for cancer therapy. Clin Epigenetics 5: 3, 2013 .

23. Feldman RJ, Sementchenko VI, Gayed M, Fraig MM and Watson DK: Pdef expression in human breast cancer is correlated with invasive potential and altered gene expression. Cancer Res 63: 4626-4631, 2003.

24. Ghadersohi A, Pan D, Fayazi Z, Hicks DG, Winston JS and Li F: Prostate-derived Ets transcription factor (PDEF) downregulates survivin expression and inhibits breast cancer cell growth in vitro and xenograft tumor formation in vivo. Breast Cancer Res Treat 102: 19-30, 2007.

25. Oettgen P, Finger E, Sun Z, et al: PDEF, a novel prostate epithelium-specific Ets transcription factor, interacts with the androgen receptor and activates prostate-specific antigen gene expression. J Biol Chem 275: 1216-1225, 2000

26. Thompson HG, Harris JW, Wold BJ, Lin F and Brody JP: p62 overexpression in breast tumors and regulation by prostatederived Ets factor in breast cancer cells. Oncogene 22: 2322-2333, 2003.

27. Zhang M, Maass N, Magit D and Sager R: Transactivation through Ets and Apl transcription sites determines the expression of the tumor-suppressing gene maspin. Cell Growth Differ 8: 179-186, 1997.

28. Zhang M, Magit D and Sager R: Expression of maspin in prostate cells is regulated by a positive Ets element and a negative hormonal responsive element site recognized by androgen receptor. Proc Natl Acad Sci USA 94: 5673-5678, 1997.

29. Gros C, Fahy J, Halby L, et al: DNA methylation inhibitors in cancer: recent and future approaches. Biochimie 94: 2280-2296, 2012.

30. Marks PW: Decitabine for acute myeloid leukemia. Expert Rev Anticancer Ther 12: 299-305, 2012.

31. Nebbioso A, Carafa V, Benedetti R and Altucci L: Trials with 'epigenetic' drugs: an update. Mol Oncol 6: 657-682, 2012. 